\title{
A Novel Fractional Fourier Transform-Based ASK-OFDM System for Underwater Acoustic Communications
}

\author{
Rami Ashri ${ }^{1}$ (D), Heba Shaban ${ }^{1, *}$ (D) and Mohamad Abou El-Nasr ${ }^{2}$ \\ 1 Department of Electronics and Communications Engineering, College of Engineering and Technology, \\ Arab Academy for Science, Technology \& Maritime Transport (AASTMT), Alexandria 1029, Egypt; \\ ramiashri@gmail.com \\ 2 Department of Computer Engineering, College of Engineering and Technology, Arab Academy for Science, \\ Technology \& Maritime Transport (AASTMT), Alexandria 1029, Egypt; mnasr@aast.edu \\ * Correspondence: hshaban@vt.edu
}

Received: 29 October 2017 ; Accepted: 6 December 2017; Published: 11 December 2017

\begin{abstract}
A key research area in wireless transmission is underwater communications. It has a vital role in applications such as underwater sensor networks (UWSNs) and disaster detection. The underwater channel is very unique as compared to other alternatives of transmission channels. It is characterized by path loss, multipath fading, Doppler spread and ambient noise. Thus, the bit error rate (BER) is increased to a large extent when compared to its counterpart of cellular communications. Acoustic signals are the current best solution for underwater communications. The use of electromagnetic or optical waves obviously entails a much higher data rate. However, they suffer from high attenuation, absorption or scattering. This paper proposes a novel fractional fast Fourier transform (FrFT) — orthogonal frequency division multiplexing (FrFT-OFDM) system for underwater acoustic (UWA) communication-which employs the amplitude shift keying (ASK) modulation technique (FrFT-ASK-OFDM). Specifically, ASK achieves a better bandwidth efficiency as compared to other commonly used modulation techniques, such as quadrature amplitude modulation (QAM) and phase shift keying (PSK). In particular, the system proposed in this article can achieve a very promising BER performance, and can reach higher data rates when compared to other systems proposed in the literature. The BER performance of the proposed system is evaluated numerically, and is compared to the corresponding $M$-ary QAM system in the UWA channel for the same channel conditions. Moreover, the performance of the proposed system is compared to the conventional fast Fourier transform (FFT)-OFDM (FFT-OFDM) system in the absence and presence of the effect of carrier frequency offset (CFO). Numerical results show that the proposed system outperforms the conventional FFT-based systems for UWA channels, even in channels dominated by CFO. Moreover, the spectral efficiency and data rate of the proposed system are approximately double the values of the corresponding conventional OFDM systems for the same parameters.
\end{abstract}

Keywords: amplitude shift keying (ASK); carrier frequency offest (CFO); fractional Fourier transform (FrFT); orthogonal frequency division multiplexing (OFDM); underwater acoustic (UWA) channels

\section{Introduction}

The research area of underwater communications has received much attention during the past years. This is motivated by the growing interest of multiple countries to develop ocean monitoring systems. These systems have applications that include environmental monitoring, oil field exploration, pollution control, seismic alerts, control of autonomous underwater vehicles (AUVs) and other military activities [1]. Acoustic signals represent the best current technology for underwater communications. 
Electromagnetic waves can propagate only at low frequencies, which in turn require large antennas and high transmission power. Furthermore, optical transmission is greatly influenced by scattering, high levels of absorption and ambient noise [1,2].

The underwater environment is a very challenging channel due to its unparalleled physical properties and specifications [3]. It is mainly characterized by path loss, multipath fading, large extent of Doppler spread and ambient noise [1,2,4]. It is also known that the UWA channel suffers from high propagation delay due to the relatively low-transmission speed $(1500 \mathrm{~m} / \mathrm{s})$, limited bandwidth, and high transmission power requirements. Moreover, different temperature degrees, salinity and pressure levels between multiple layers of sea water, add to the complexity of the UWA channel $[1,2]$.

Orthogonal Frequency Division Multiplexing (OFDM) has dominated in recent underwater wireless communication systems. This is motivated by the success of OFDM in cellular communications. OFDM is characterized by its high spectral efficiency and low equalization complexity in the presence of frequency selective channels. However, OFDM suffers from the effect of Doppler spread due to the movement of the transmitter or the receiver, which consequently creates non-uniform carrier frequency offset (CFO). This poses great complexity for the channel equalizer and receiver, which in turn limits any fundamental improvement in terms of data rate. The commonly used digital modulation techniques with OFDM include quadrature amplitude modulation (QAM) and phase shift keying (PSK). The application of amplitude shift keying (ASK) to OFDM in cellular systems has been proposed in [5], and its performance was evaluated in the additive white Gaussian noise (AWGN) channel in [6], and in [7] the performance was evaluated in the Rayleigh fading channel. ASK-OFDM requires $1 / 2 T$ as the minimum frequency spacing for maintaining the orthogonality between sub-carriers, which is half the spacing required by PSK-OFDM and QAM-OFDM schemes. This bandwidth saving is traded for a higher peak-average-power-ratio (PAPR). However, there are many solutions which exist in the literature that reduce PAPR [7].

Generally, OFDM is efficiently implemented by fast Fourier transform (FFT), and it is effective in the underwater communication channel since it transforms the frequency selective channel into several narrow flat fading sub-channels. This in turn makes the system robust against inter-symbol-interference (ISI) induced by large channel delay spread. Though OFDM is better than single carrier transmission techniques, it still cannot be used for long range transmissions over UWA channels due to its poor BER performance. Similar to wireless communications, OFDM-based communications for UWA channels have been extensively studied in the literature. These studies included channel charectarization and measurements [8-10], system design and performance evaluation [11,12], synchronization and channel estimation [13-15], inter-carrier-interference (ICI) cancellation [16], and the investigation of multiple-input-multiple-output (MIMO)-OFDM [17]. The commonly used modulation schemes with OFDM systems in UWA channels are Quadrature Phase Shift Keying (QPSK), equivalent to 4-QAM, and 16-QAM [11,12,18,19].

One method to improve the performance of OFDM systems is to replace the inverse fast Fourier transform (IFFT) and FFT by the FrFT; this enhances the BER performance, and thus makes it suitable for longer range communications in UWA channels. FFT-based OFDM is limited to the rotation of the time-frequency plane by multiples of $(\pi / 2)$ only. On the other hand, FrFT-based OFDM allows for a rotation of the signal by any fractional angle $(\alpha)$, and thus gives it one more degrees of freedom. Therefore, it can reduce the BER to a large extent. The FrFT is similar to Fourier transform when $\alpha=\pi / 2$. The FrFT-OFDM system has been recently proposed for wireless communications, and was investigated in Rayleigh fading channels assuming binary phase shift keying (BPSK) modulation [20]. Studies of FrFT-OFDM for UWA communications are still in their early stages. In [21,22], channel estimation was investigated, and in [23] a more complicated form of FrFT was considered for channel equalization. In $[24,25]$, the authors proposed pulse position modulation (PPM) based FrFT-OFDM for UWA channels. The suggested technique has a low BER performance and does not utilize the scarce bandwidth, thus resulting in a poor bandwidth efficiency when compared to the ASK FrFT-OFDM system proposed in this paper. Also, even though the rake receiver proposed in [24] 
to eliminate the multipath effect was complex, the system suffered from inter-symbol-interference (ISI). In [26], we investigated the performance of the FrFT-OFDM system for UWA communications with QAM modulation.

This article proposes a novel FrFT-ASK-OFDM system for frequency selective underwater acoustic (UWA) channels. The proposed system has the potential to greatly enhance the bit error rate (BER) performance as compared to conventional OFDM systems. This enhancement is traded for higher peak-average-power-ratio (PAPR). It also compares the BER performance of the proposed system to the conventional OFDM systems with $M$-ary QAM modulation in UWA channels. Moreover, it studies the effect of CFO on the BER performance in UWA channels.

The remainder of this article is organized as follows. An overview of conventional OFDM systems with ASK modulation is given in Section 2. The FrFT is briefly introduced in Section 3. A summary of the UWA channel is given in Section 4. Section 5 introduces the proposed system model. Then, Section 6 provides the simulation results and performance evaluation of the $M$-ary ASK FrFT-OFDM system under the effect of CFO over the UWA channel. Finally, conclusions and future work are given in Section 7.

\section{Overview of ASK-OFDM}

The PSK and QAM schemes are the most common modulation techniques used with OFDM. More specifically, QPSK and 16-QAM schemes have been widely used. Both methods use $1 / T$ as the separation distance between the subcarriers to maintain orthogonality among them. On the other hand, ASK requires only $1 / 2 T$ as the minimum frequency separation, since the subcarriers differ only in amplitudes and frequencies, while their phases are the same $(0, \pi / 2$ or $\pi)$ [4-6]. Therefore, ASK-OFDM attains better bandwidth efficiency. Furthermore, the ASK-OFDM system is not as sophisticated as conventional OFDM systems that use QAM or PSK. M-ary ASK-OFDM signal as introduced in [5]:

$$
s(t)=\sum_{k=0}^{N-1} A_{k} \cos \left(2 \pi f_{k} t\right)
$$

where, $A_{k}$ is the amplitude of the $M$-ary ASK-OFDM signal, $M$ is the modulation order, $N$ is the number of subcarriers and $f_{k}=k / 2 T$ is the subcarrier frequency. The subcarrier frequency separation is $1 / 2 T$.

Figures 1 and 2 show the spectra of OFDM subcarriers with 1/T spacing for PSK and QAM, and $1 / 2 T$ for ASK, respectively. It is obvious from the figures that the bandwidth saving ratio of ASK-OFDM compared to PSK and QAM-OFDM as given by [5] is:

$$
\text { BandwidthSaving }=\frac{(2(N+1))}{(N+3)}
$$

ASK on the other hand has lower power efficiency when compared to PSK or QAM for the same modulation order $M$. The increase in PAPR compared to PSK-OFDM is expressed as [5]:

$$
P A P R_{\mathrm{degr}}=\frac{3(M-1)}{M+1}
$$

However, ASK is still favorable for implementation with the OFDM system since there are techniques in the literature which reduce PAPR. One dynamic solution to solve the high PAPR problem is through coding. There are some preferable coding approaches dedicated to the PAPR issue discussed in [7]. 


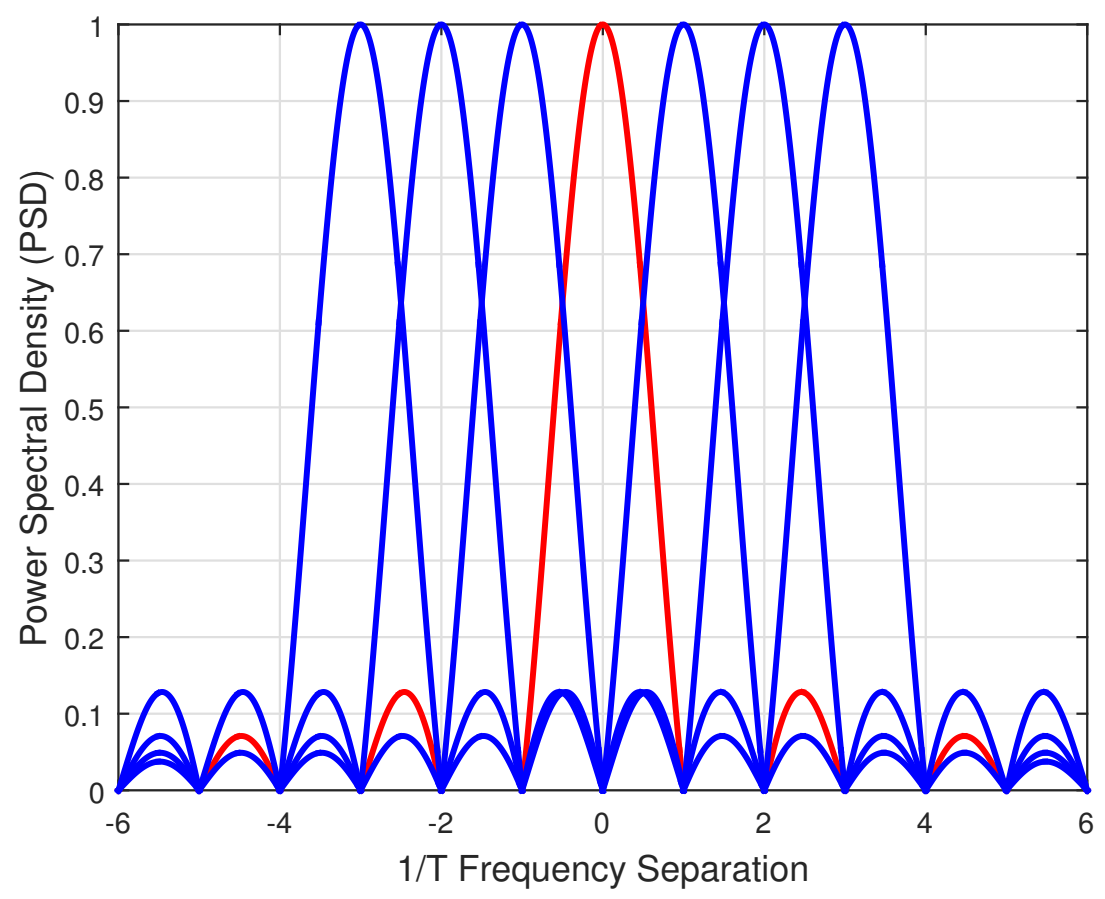

Figure 1. Spectrum of an OFDM signal with $N$ subcarriers. Subcarriers of $M$-ary QAM OFDM or M-ary PSK-OFDM with spacing of $1 / T$.

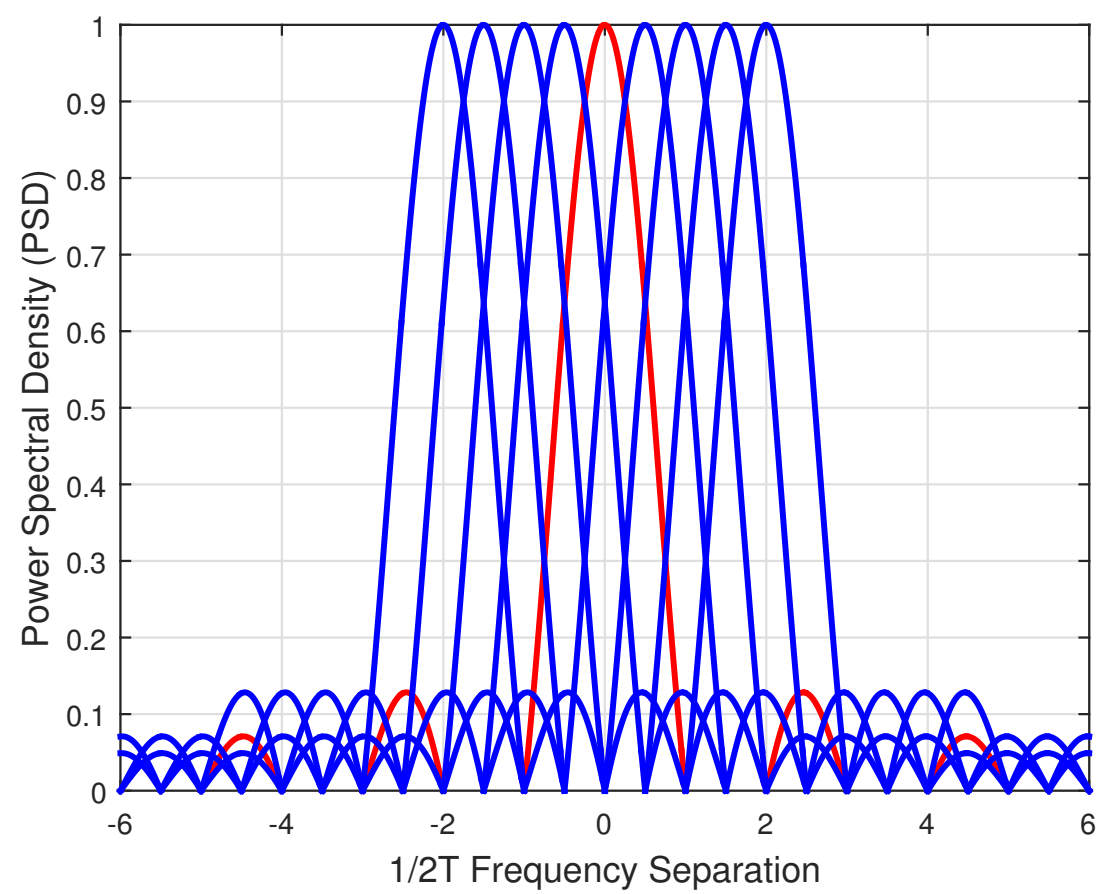

Figure 2. Spectrum of an OFDM signal with $N$ subcarriers. Subcarriers of $M$-ary ASK-OFDM with spacing of $1 / 2 T$.

\section{Fractional Fourier Transform (FrFT)}

The theory of FrFT was first introduced in mathematics at the late 1980s and early 1990s [27]. It is considered as a comprehensive standard of the Fourier transform and explicated as an alternation of the rotation angle in the time-frequency analysis [28]. The conventional Fourier transform is a rotation 
of time axis at a defined angle of $\pi / 2$ with respect to the frequency axis; however, the FrFT allows the time axis of the signal to be rotated at any fractional angle $(\alpha)$ with respect to the frequency axis, that is establishing new time and fractional domain axes ( $\alpha$ domain). Fractional Fourier transform is confined to the application of chirp signals only, which are linear frequency modulation (LFM) signals. The FrFT of signal $x(t)$ as defined in $[24,29,30]$ :

$$
X_{m}=\left\{F^{m}[x(t)]\right\}(u)=\int_{-\infty}^{\infty} x(t) H_{m}(t, u) d t
$$

where $F^{m}$ is the FrFT operator, $m$ denotes a real integer and $H_{m}(t, u)$ is the FrFT kernel, which is given as $[24,29,30]$ :

$$
H_{m}(t, u)=\left\{\begin{array}{c}
\sqrt{\frac{(1-j \cot \alpha)}{2 \pi}} \exp \left(j\left(\frac{1}{2} t^{2} \cot \alpha-u t \csc \alpha+\frac{1}{2} u^{2} \cot \alpha\right)\right), \propto \neq n \pi \\
\delta\left(u-(-1)^{n} t\right), \propto=n \pi
\end{array}\right.
$$

where $\alpha=m \pi / 2$ is the rotation angle of the FrFT. Thus, Equation (4) can be explicated as [24,29,30]:

$$
X_{m}=\left\{F^{m}[x(t)]\right\}(u)=\left\{\begin{array}{c}
\sqrt{\frac{1-j \cot \alpha}{2 \pi}} \int_{-\infty}^{\infty} \exp \left(j \frac{u^{2}+t^{2}}{2} \cot \propto-j u t \csc \propto\right) x(t) d t, \propto \neq n \pi \\
x\left((-1)^{n} t\right), \propto=n \pi
\end{array}\right.
$$

The corresponding inverse fractional Fourier transform (IFrFT) as defined in [29] is given by:

$$
x(t)=\int_{-\infty}^{\infty} X_{m}(u) H_{-m}(t, u) d u
$$

Equation (7) manifests that the signal $x(t)$ is realized as decomposition to a basis formed by the ortho-normal chirp signals in the $\alpha$-domain.

The authors in [31-34] explained the implementation of discrete fractional Fourier transform (DFrFT), which falls into three categories namely, eigenvalue decomposition, discrete sample, and linear combination. The authors of this article refer to the decomposition algorithm used in [29], which is based on the discrete algorithm submitted by Ozaktas in [32,33]. In the Ozaktas model, the FrFT is decomposed into two multiplications and one convolution with the chirp signal. The advantage of this decomposition algorithm is the high calculation accuracy with low computational complexity. It is worth noting that the complexity of DFrFT is similar to DFT [23].

\section{Underwater Acoustic Channel Model}

The unparalleled characteristics of the underwater acoustic (UWA) channel are presented in this section with a highlight on the major drawbacks and limitations of signal propagation in this medium. We assume a time-varying channel model with impulse response as [9,13]:

$$
h(\tau, t)=\sum_{l} h_{l}(t) \delta\left(\tau-\tau_{l}(t)\right)
$$

where $h_{l}(t)$ is the $l$ th path amplitude, and $\tau_{l}(t)$ is the time-varying path delay.

\subsection{Path Loss}

The path loss has a great effect on the wireless communication channel. The spreading and absorption of the signal are the main causes of path loss in the UWA channel. The total path loss in $\mathrm{dB}$ as introduced in [1]:

$$
\text { PathLoss }=10 s \log _{10}(d)+10 d \log _{10}(a(f))
$$


where $d$ represents the distance between the transmitter and receiver, $a(f)$ is the absorption coefficient and $s$ denotes the spreading factor. The absorption coefficient $a(f)$ is dependent on frequency of propagation, temperature, and pressure. The spreading factor is based on the geometry of propagation. For a practical UWA channel, the spreading factor $s$ is a hybrid of spherical and cylindrical geometric spreading, with a value of $s=1.5$ [1].

\subsection{Fading}

Fading is the level of the received power, which oscillates due to the nature of multipath propagation in an underwater environment. In shallow water, fading is a result of wave reflection from the surface and bottom, while in deep ocean zones, it is due to a natural phenomenon known as refraction. On the one hand, multipath fading causes the transmitted signal to travel through many paths to reach the receiver with different delay spreads. These delays overlap each other and cause inter-symbol interference (ISI), which in turn points towards a frequency-selective channel. Sound travels at a velocity of $1500 \mathrm{~m} / \mathrm{s}$ underwater [1]. This introduces additional delay spread. The approximate delay spread of typical underwater channels is $10 \mathrm{~ms}$, but sometimes delay spread can be as large as 50 to $100 \mathrm{~ms}$.

On the other hand, the underwater acoustic environment is subject to surface scattering as well as internal waves, which introduces the time selectivity of the channel model. Doppler spreads are dominated by sea surface conditions and transmitter or receiver motion. The receiver design of the UWA-OFDM system has to employ effective techniques to compensate the Doppler spread for proper operation.

\subsection{Noise Model}

Common sources of ambient noise in underwater acoustic channels include shipping activities, rain fall, seismic events and sounds generated by aquatic animals. The Wenz model classifies the noise sources into four main categories, according to the frequency spectrum. In the frequency range below $10 \mathrm{~Hz}$, the main source of noise is turbulence due to high waves. In the frequency range of $10 \mathrm{~Hz}-100 \mathrm{~Hz}$, noise is caused by shipping activities. Surface movement due to wind and waves is the primary reason of noise in the frequency range between $100 \mathrm{~Hz}$ and $100 \mathrm{kHz}$. Furthermore, at frequencies higher than $100 \mathrm{kHz}$, electronic thermal noise is the dominating factor. The total ambient noise level is given by adding the level sum of the participating noise factors.

\section{System Model}

This section presents the proposed FrFT-based OFDM system for underwater communication. Figure 3 presents a block diagram of the system model. This article considers the Rician fading channel model, as it was found to provide a good match for underwater channels $[8,9]$. 


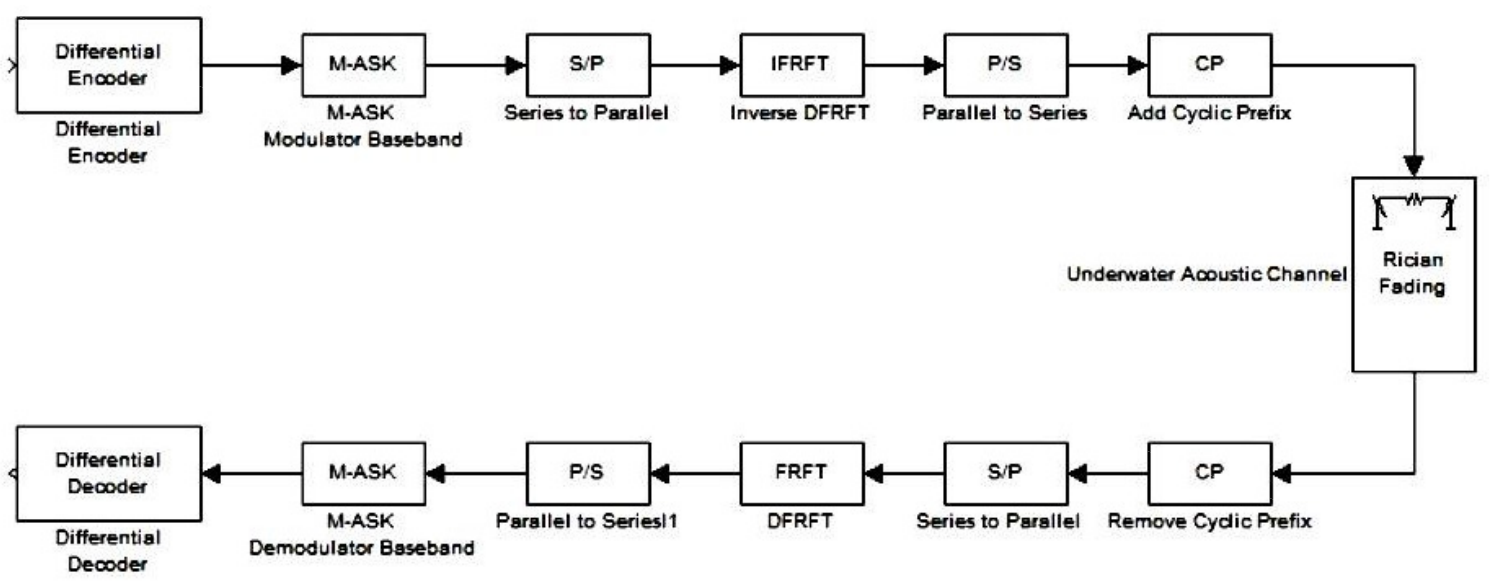

Figure 3. ASK FrFT-OFDM System model. A schematic diagram for ASK-OFDM based on FrFT for UWA communication.

The structure of the system is similar to the ordinary OFDM system, where the IFFT and FFT blocks are replaced by IDFrFT and DFrFT blocks. The system uses a $M$-ary ASK modulator/demodulator. An advantage of $M$-ary FrFT-ASK-OFDM is that it achieves high power and saves bandwidth. That is, it provides a good application for the UWA channel which is bandwidth-limited. The cyclic prefix (CP) is added to the transmitted signal in order to suppress the ISI, which is due to multipath fading. OFDM suffers greatly from the effect of CFO which induces inter carrier interference (ICI). Furthermore, the effect of CFO becomes more severe as the number of subcarriers increases and BER increases to a large extent. However, this is not the case in FrFT-based OFDM, where ICI can be extremely suppressed by using IFrFT and FrFT instead of IFFT and FFT.

Reported parameters for OFDM systems presented in the literature based on actual applications were not fixed. A summary of the range of common parameters is as follows. Carrier frequencies ranged from $8 \mathrm{kHz}$ to $24 \mathrm{kHz}$ and bandwidths ranged from $3 \mathrm{kHz}$ up to $50 \mathrm{kHz}$. The number of subcarriers $N$ reported in the literature varied, and values were up to 2048 sub-carriers. Sub-carrier spacings were on the order of tens of Hertz up to hundred Hertz [13,16-18].

\section{Spectral Efficiency and Data Rate}

Two important parameters considered for performance evaluation of communication systems are the spectral efficiency and data rate particularly for UWA, where the data rate represents a great challenge due to the limited bandwidth.

The bandwidth for QAM/PSK systems is $B_{Q}=\frac{N+1}{T}$, where for ASK OFDM systems the bandwidth is $B_{A}=\frac{N+3}{2 T}$ [5]. For QAM/PSK OFDM systems, the spectral efficiency is calculated as $\alpha_{Q}[18]$ :

$$
\alpha_{Q}=\frac{T}{T+T_{g}} \frac{N_{d}}{N+1} \log _{2}(M)
$$

where $T_{g}$ is the guard time, and $N_{d}$ is the number of effective data subcarriers. For ASK-OFDM, the spectral efficiency is given by $\alpha_{A}$ :

$$
\alpha_{A}=\frac{2 T}{T+T_{g}} \frac{N_{d}}{N+3} \log _{2}(M)
$$

Thus, the data rate for QAM/PSK OFDM systems is calculated as $\alpha_{Q} B$, and for ASK-OFDM is $\alpha_{A} B$. As can be seen, for the same parameters and modulation order, the spectral efficiency of ASK-OFDM will be approximately double the corresponding spectral efficiency of QAM/PSK OFDM systems. 


\section{Numerical Results}

This section presents numerical results for the proposed system. We investigate both the ordinary FFT-OFDM well studied for UWA systems with QPSK (4-QAM) and 16 QAM, and compare it to the novel FrFT-OFDM systems with the following parameters: $N=64$ subcarriers; cyclic prefix $(\mathrm{nCP})=8$, delay taps (nTap) $=2$ and modulation scheme is $M$-ary ASK. The propagation environment is a frequency selective UWA channel in the presence of CFO, where the Rician $k$-factor is in the order of 10 for UWA channel model. Also, a wide range of fractional order $(\alpha)$ has been tried to verify the simulation results; specifically, $\alpha=1.0$ (FFT), $\alpha=1.576, \alpha=1.580, \alpha=1.582, \alpha=1.584$ and $\alpha=1.62$. Figure 4 shows a BER performance comparison of a FrFT-based OFDM system assuming $M$-ary ASK and $M$-ary QAM modulation techniques in UWA channel with $\mathrm{CFO}=0.05$. The plot shows that both 2-ary FrFT-ASK-OFDM and 4-ary FrFT-QAM-OFDM have identical BER performance. Similarly, 4-ary FrFT-ASK-OFDM has similar BER performance as 16-ary FrFT-QAM-OFDM. Thus, $\sqrt{(M)}$-ary FrFT-ASK-OFDM achieves comparable BER performance to their counterpart of M-ary FrFT-QAM-OFDM. All simulations have been tested for 10,000 samples.

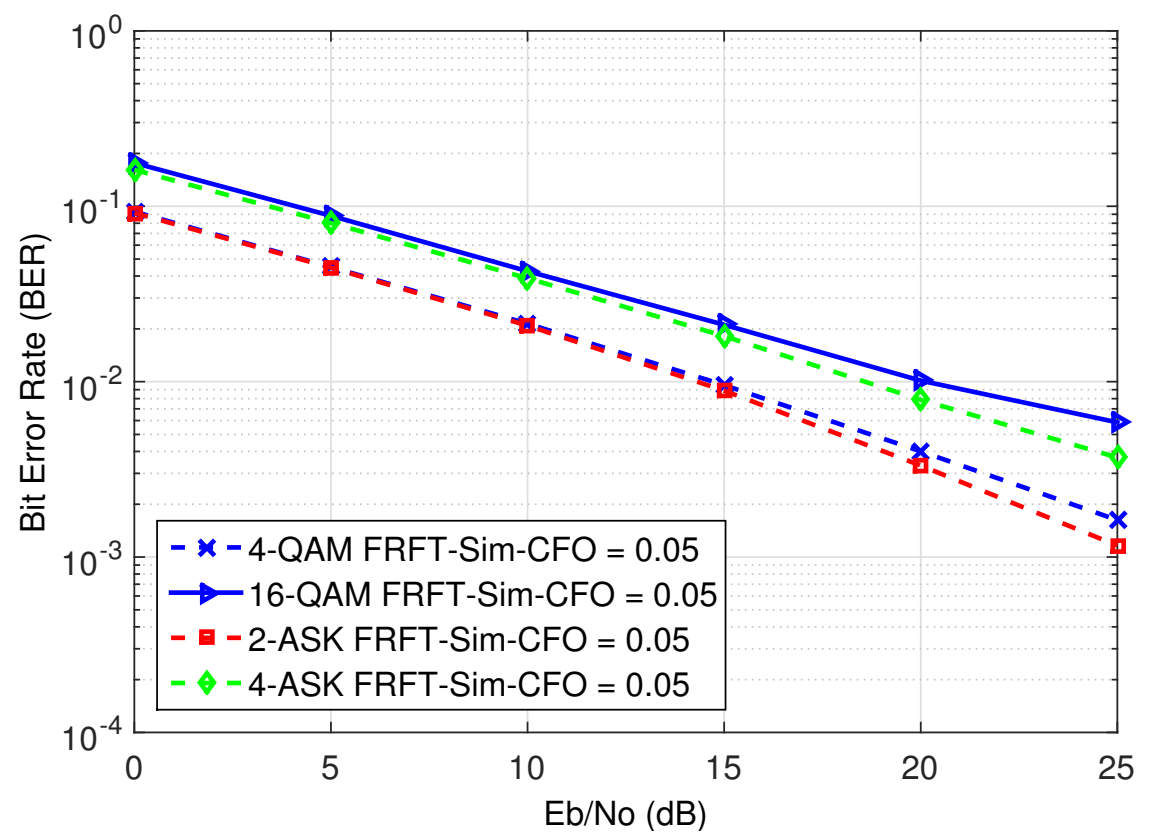

Figure 4. BER performance comparison of M-ary FrFT-ASK-OFDM and M-ary FrFT-QAM-OFDM in the frequency-selective UWA channel with $\mathrm{CFO}=0.05$.

Figure 5 shows a BER comparison of 2-ASK FrFT and the FFT-based OFDM system over the frequency selective UWA channel in the presence of different CFOs. As can be seen, the proposed FrFT-ASK-OFDM system outperforms the corresponding FFT-ASK-OFDM in the UWA channel.

Figure 6 shows the BER performance of the FrFT-OFDM system at different values of fractional order $(\alpha)$ in the same channel conditions with $\mathrm{CFO}=0.15$. It is visible that at $\alpha=\pi / 2$, both the FrFT-ASK-OFDM system and the FFT-ASK-OFDM system behave in an identical manner. Also, the minimum BER performance is achieved for a value of $\alpha=1.584$ and the performance degrades with changing this value. 


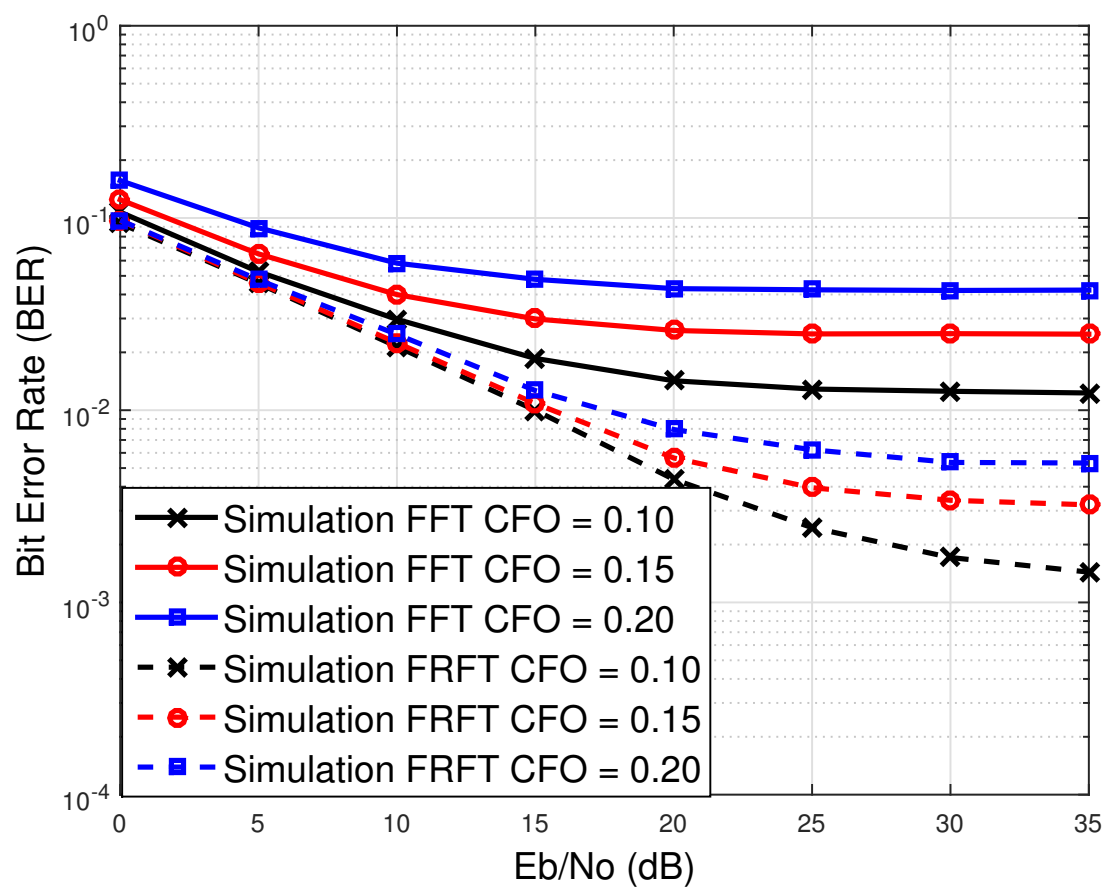

Figure 5. BER for 2-ASK FrFT-based OFDM over the frequency-selective UWA channel with CFO = 0.1, 0.15 , and 0.2 .

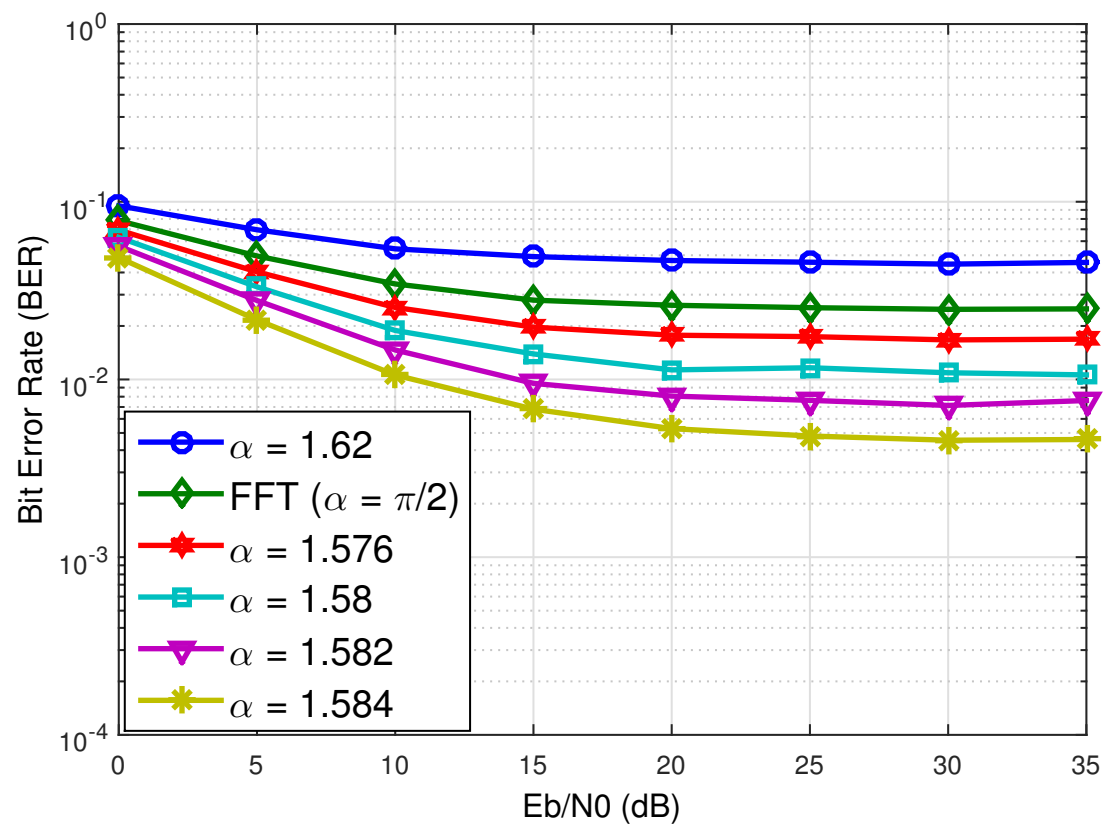

Figure 6. BER for 2-ASK FrFT-based OFDM over the frequency-selective UWA channel with CFO $=0.15$ for different values of $\alpha$.

Figure 7 depicts the BER performance comparison of 2-ASK, 4-ASK, 8-ASK and 16-ASK FrFT-OFDM in the UWA channel with $\mathrm{CFO}=0.05$, which shows the tradeoff between BER performance and data rate. 


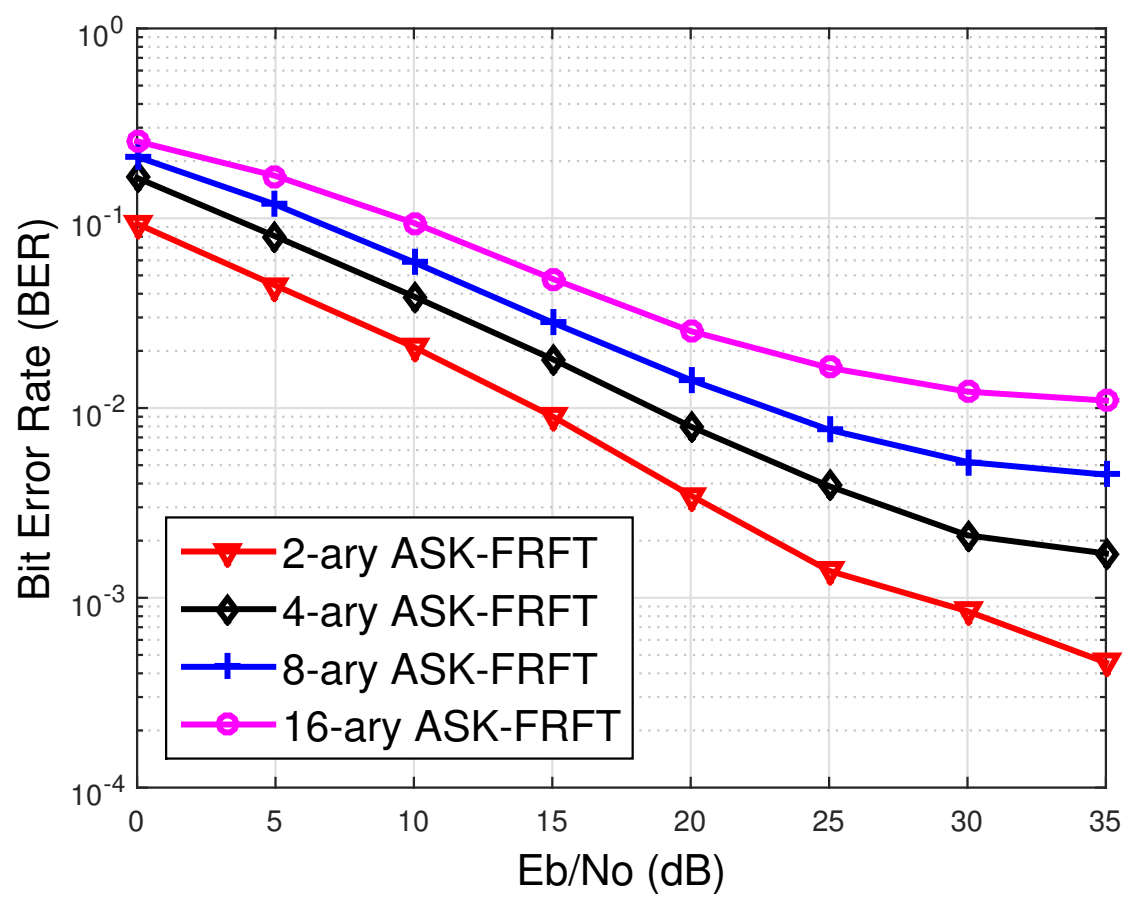

Figure 7. BER performance of $M$-ary FrFT-ASK-OFDM in the frequency-selective UWA channel with $\mathrm{CFO}=0.05$.

A summary of the achievable BER for QPSK, 16-QAM, 4-ASK and 16-ASK for different SNRs is given in Table 1 for $\mathrm{CFO}=0.05$. As can be seen, the BER ranges from 0.001 to 0.094. Recent BER results presented in the literature ranged from 0.005 to 0.25 as in [35].

Table 1. BER comparison of OFDM systems under investigation at different SNRs for CFO = 0.05.

\begin{tabular}{lcccc}
\hline & QPSK & 16-QAM & 4-ASK & 16-ASK \\
\hline SNR $=10 \mathrm{~dB}$ & 0.021 & 0.039 & 0.039 & 0.094 \\
$\mathrm{SNR}=17 \mathrm{~dB}$ & 0.006 & 0.013 & 0.013 & 0.037 \\
$\mathrm{SnR}=25 \mathrm{~dB}$ & 0.001 & 0.004 & 0.004 & 0.016 \\
\hline
\end{tabular}

We aimed to calculate the spectral efficiency and data rate based on realistic parameters reported in the literature, and compare them for the cases under investigation. For $T=85.33 \mathrm{~ms}, T_{g}=25 \mathrm{~ms}$, $N_{d}=672$, and $N=1024$, the spectral efficiencies and data rates are as follows. For QPSK, the spectral efficiency $\alpha_{Q}=1.014$, and for 16-QAM $\alpha_{Q}=2.02821$. The corresponding spectral efficiencies for 4-ASK and 16-ASK are 2.024 and 4.0485, respectively. The corresponding data rates assuming long range $B=5 \mathrm{kHz}$, medium range $B=10 \mathrm{kHz}$, and small range $B=50 \mathrm{kHz}$ are summarized in Table 2 . As can be seen, for the same modulation order, ASK-OFDM achieves approximately double the data rate compared to QAM-OFDM.

Table 2. Data rates for OFDM systems under investigation.

\begin{tabular}{ccccc}
\hline & QPSK & 16-QAM & 4-ASK & 16-ASK \\
\hline Long range & $5.07 \mathrm{kbps}$ & $10.14 \mathrm{kbps}$ & $10.12 \mathrm{kbps}$ & $20.24 \mathrm{kbps}$ \\
Medium range & $10.14 \mathrm{kbps}$ & $20.28 \mathrm{kbps}$ & $20.24 \mathrm{kbps}$ & $40.48 \mathrm{kbps}$ \\
Short range & $50.7 \mathrm{kbps}$ & $101.41 \mathrm{kbps}$ & $101.2 \mathrm{kbps}$ & $202.425 \mathrm{kbps}$ \\
\hline
\end{tabular}




\section{Conclusions and Future Work}

In this article, the authors proposed a novel FrFT-ASK-OFDM system for bandwidth-efficient, high data rate UWA communication. The proposed system employed the ASK modulation scheme, which was shown to be highly recommended in an underwater environment since it efficiently utilizes the limited bandwidth, which is not the case for PSK or QAM. The proposed system also employed FrFT with OFDM, which has the advantage of reducing the BER to a large extent in the absence and presence of CFO. Numerical results showed that the proposed system is very suitable for application in an underwater environment. This is due to its bandwidth saving and great BER performance in a channel characterized by scarce bandwidth, multipath fading and ICI due to Doppler spread. In particular, the system model proposed in this article can achieve a very promising BER performance and can reach higher data rates when compared to other system in the literature.

Regarding future work, even though the ASK modulation achieves a better bandwidth efficiency in the UWA channel when combined with FrFT as compared to commonly used modulation schemes, the BER of un-coded OFDM systems is relatively high. However, the ASK FrFT-OFDM is very promising in UWA channels, and can be combined with some other techniques to enhance the BER. In addition, the PAPR issue should be addressed carefully. Coding is one of the techniques that is mentioned in this article as an enhancement for power efficiency, but this is not within the main scope of this article. The literature is full of techniques and algorithms to reduce PAPR at the expense of more system complexity. However, these approaches will require further investigation and improvements to be compatible with the UWA communication channel. Moreover, theoretical modeling and physical experiments based on field tests will be addressed in the future to support and emphasize the simulation results presented in this study. More specifically, simulations based on Watermark benchmark software will be carried out in future work [36].

Author Contributions: Heba Shaban proposed the idea. Rami Ashri and Heba Shaban conceived and designed the simulations, analyzed the data, and wrote the paper. Heba Shaban and Mohamad Abou El-Nasr reviewed the paper and simulation results.

Conflicts of Interest: The authors declare no conflict of interest.

\section{References}

1. Al-Dharrab, S.; Uysal, M.; Duman, T.M. Cooperative Underwater Acoustic Communications [Accepted From Open Call]. IEEE Commun. Mag. 2013, 51, 146-153. doi:10.1109/MCOM.2013.6553691.

2. Rehan, K.; Qiao, G. A Survey of Underwater Acoustic Communication and Networking Techniques. Res. J. Appl. Sci. Eng. Technol. 2013, 5, 1778-789.

3. Paul, J.; Gendron, Z.H.M. Shallow Water Acoustic Response and Platform Motion Modeling via a Hierarchical Gaussian Mixture Model. J. Acoust. Soc. Am. 2016, 139, 1923-1937. doi:10.1121/1.4943552.

4. Che, X.; Wells, I.; Dickers, G.; Kear, P.; Gong, X. Re-Evaluation of RF Electromagnetic Communication in Underwater Sensor Networks. IEEE Commun. Mag. 2010, 48, 143-151, doi:10.1109/MCOM.2010.5673085.

5. Xiong, F. M-ary Amplitude Shift Keying OFDM System. IEEE Trans. Commun. 2003, 51, 1638-1642. doi:10.1109/TCOMM.2003.818103.

6. Nasr, M.A.; Shaban, H.A.; Mangoud, M.A. Coded 4-PAM OFDM for High Rate Data Links. In Innovations and Advanced Techniques in Computer and Information Sciences and Engineering; Sobh, T., Ed.; Springer: Dordrecht, The Netherlands, 2007; pp. 553-556. doi:10.1007/978-1-4020-6268-1_97.

7. Shaban, H.; Khedr, M.; Hamed, K. M-Ary Amplitude Shift Keying-Orthogonal Frequency Division Multiplexing for High Data-Rate Wireless Communications Over Rayleigh Fading Channels in Presence of Carrier Frequency Offset. Adv. Sci. Lett. 2014, 20, 418-421, doi:10.1166/asl.2014.5321.

8. Clemente, M.C.; Paris, J.F. Closed-Form Statistics for Sum of Squared Rician Shadowed Variates and its Application. Electron. Lett. 2014, 50, 120-121. doi:10.1049/el.2013.0969.

9. Radosevic, A.; Proakis, J.G.; Stojanovic, M. Statistical Characterization and Capacity of Shallow Water Acoustic channels. In Proceedings of the OCEANS 2009-EUROPE, Bremen, Germany, 11-14 May 2009; pp. 1-8. 
10. Stojanovic, M.; Preisig, J. Underwater Acoustic Communication Channels: Propagation Models and Statistical Characterization. IEEE Commun. Mag. 2009, 47, 84-89. doi:10.1109/MCOM.2009.4752682.

11. Bejjani, E.; Belfiore, J.C. Multicarrier Coherent Communications for the Underwater Acoustic Channel. In Proceedings of the Prospects for the 21st Century OCEANS'96 Conference, Fort Lauderdale, FL, USA, 23-26 September 1996; volume 3, pp. 1125-1130.

12. Lam, W.K.; Ormondroyd, R.F. A Coherent COFDM Modulation System for a Time-Varying Frequency-Selective Underwater Acoustic Channel. In Proceedings of the Seventh International Conference on Electronic Engineering in Oceanography-Technology Transfer from Research to Industry, Southampton, UK, 23-25 June 1997; pp. 198-203.

13. Kim, B.C.; Lu, I.T. Parameter Study of OFDM Underwater Communications System. In Proceedings of the OCEANS 2000 MTS/IEEE Conference and Exhibition, Providence, RI, USA, 11-14 September 2000; Volume 2, pp. 1251-1255.

14. Kang, T.; Iltis, R.A. Iterative Carrier Frequency Offset and Channel Estimation for Underwater Acoustic OFDM Systems. IEEE J. Sel. Areas Commun. 2008, 26, 1650-1661. doi:10.1109/JSAC.2008.081205.

15. Stojanovic, M. OFDM for Underwater Acoustic Communications: Adaptive Synchronization and Sparse Channel Estimation. In Proceedings of the 2008 IEEE International Conference on Acoustics, Speech and Signal Processing, Las Vegas, NV, USA, 31 March-4 April 2008 ; pp. 5288-5291.

16. Kuai, X.; Sun, H.; Zhou, S.; Cheng, E. Impulsive Noise Mitigation in Underwater Acoustic OFDM Systems. IEEE Trans. Veh. Technol. 2016, 65, 8190-8202. doi:10.1109/TVT.2016.2516539.

17. Li, B.; Huang, J.; Zhou, S.; Ball, K.; Stojanovic, M.; Freitag, L.; Willett, P. MIMO-OFDM for High-Rate Underwater Acoustic Communications. IEEE J. Ocean. Eng. 2009, 34, 634-644. doi:10.1109/JOE.2009.2032005.

18. Li, B.; Zhou, S.; Huang, J.; Willett, P. Scalable OFDM Design for Underwater Acoustic Communications. In Proceedings of the 2008 IEEE International Conference on Acoustics, Speech and Signal Processing, Las Vegas, NV, USA, 31 March-4 April 2008; pp. 5304-5307.

19. Songzuo, L.; Lu, M.; Hui, L.; Tingting, C.; Gang, Q. Design and Implementation of OFDM Underwater Acoustic Communication Algorithm Based on OMAP-L138. In Proceedings of the Proceedings of the International Conference on Underwater Networks \& Systems, Rome, Italy, 12-14 November 2014; pp. 12:1-12:5.

20. Kumari, S.; Rai, S.K.; Kumar, A.; Joshi, H.D.; Singh, A.K.; Saxena, R. Exact BER Analysis of FRFT-OFDM System Over Frequency Selective Rayleigh Fading Channel with CFO. Electron. Lett. 2013, 49, 1299-1301. doi:10.1049/el.2013.0980.

21. Chen, Y.; Clemente, C.; Soraghan, J.; Weiss, S. Fractional Fourier Based Sparse Channel Estimation for Multicarrier Underwater Acoustic Communication System. In Proceedings of the 2016 Sensor Signal Processing for Defence (SSPD), Edinburgh, UK, 22-23 September 2016; pp. 1-5.

22. Chen, Y.; Clamente, C.; Soraghan, J.; Weiss, S. Fractional Cosine Transform (FRCT)-Turbo Based OFDM for Underwater Acoustic Communication. In Proceedings of the Sensor Signal Processing for Defence (SSPD), Edinburgh, UK, 9-10 September 2015; pp. 1-5.

23. Chen, Y.; Clemente, C.; Soraghan, J.J.; Weiss, S. Partial Fractional Fourier Transform (PFRFT)-OFDM for Underwater Acoustic Communication. In Proceedings of the 2015 23rd European Signal Processing Conference (EUSIPCO), Nice, France, 31 August-4 September 2015; pp. 364-368.

24. Chen, Y.; Cai, P.; Wang, Y. A New Underwater Acoustic Communication System Based on Fractional Fourier Transform. In Proceedings of the 2010 IEEE International Conference on Information and Automation (ICIA), Harbin, China, 20-23 July 2010; pp. 413-418.

25. Zhu, Z.; Chen, Y.; Cai, P. A Novel Underwater Acoustic FRFT-OFDM Communication System with VTRM Technology. Int. J. Adv. Comput. Technol. 2012, 4, 82-92. doi:10.4156/ijact.vol4.issue16.10.

26. Ashri, R.M.; Shaban, H.A.; El-Nasr, M.A. BER of FRFT-Based OFDM System for Underwater Wireless Communication. In Proceedings of the 2016 33rd National Radio Science Conference (NRSC), Aswan, Egypt, 22-25 February 2016; pp. 266-273.

27. Martone, M. A Multicarrier System Based on the Fractional Fourier Transform for Time-Frequency-Selective Channels. IEEE Trans. Commun. 2001, 49, 1011-1020. doi:10.1109/26.930631.

28. Chen, E.; Tao, R.; Meng, X. The OFDM System Based on the Fractional Fourier Transform. In Proceedings of the First International Conference on Innovative Computing, Information and Control, Beijing, China, 30 August-1 September 2006; Volume 3, pp. 14-17. 
29. Chen, Y.; Cai, P.; Wang, Y. Research on FRFT-PPM Underwater Acoustic Communication System. In Proceedings of the 2010 3rd International Congress on Image and Signal Processing (CISP), Yantai, China, 16-18 October 2010; Volume 9, pp. 4427-4431.

30. Chen, X.; Guan, J.; He, Y.; Zhang, J. Detection of Low Observable Moving Target in Sea Clutter via Fractal Characteristics in Fractional Fourier Transform Domain. IET Radar Sonar Navig. 2013, 7, 635-651. doi:10.1049/iet-rsn.2012.0116.

31. Candan, C.; Kutay, M.A.; Ozaktas, H.M. The Discrete Fractional Fourier Transform. IEEE Trans. Signal Process. 2000, 48, 1329-1337. doi:10.1109/78.839980.

32. Ozaktas, H.M.; Arikan, O.; Kutay, M.A.; Bozdagt, G. Digital Computation of The Fractional Fourier Transform. IEEE Trans. Signal Process. 1996, 44, 2141-2150. doi:10.1109/78.536672.

33. Pei, S.C.; Ding, J.J. Closed-Form Discrete Fractional and Affine Fourier Transforms. IEEE Trans. Signal Process. 2000, 48, 1338-1353. doi:10.1109/78.839981.

34. Santhanam, B.; McClellan, J.H. The Discrete Rotational Fourier Transform. IEEE Trans. Signal Process. 1996, 44, 994-998. doi:10.1109/78.492554.

35. Zhao, A.; Zeng, C.; Hui, J.; Ma, L.; Bi, X. Experimental Demonstration of Long-Range Underwater Acoustic Communication Using a Vertical Sensor Array. Sensors 2017, 17, 1516. doi:10.3390/s17071516.

36. Walree, P.V.; Otnes, R.; Jenserud, T. Watermark: A Realistic Benchmark for Underwater Acoustic Modems. In Proceedings of the 2016 IEEE Third Underwater Communications and Networking Conference (UComms), Lerici, Italy, 30 August-1 September 2016; pp. 1-4.

(C) 2017 by the authors. Licensee MDPI, Basel, Switzerland. This article is an open access article distributed under the terms and conditions of the Creative Commons Attribution (CC BY) license (http:/ / creativecommons.org/licenses/by/4.0/). 